\title{
Producing Ni-base Superalloys Single Crystal by Selective Electron Beam Melting
}

Edouard Chauvet ${ }^{1}$, Catherine Tassin, Jean-Jacques Blandin ${ }^{1}$, Rémy Dendievel ${ }^{1}$, Guilhem Martin ${ }^{1 \#}$

1. Univ. Grenoble Alpes, CNRS, Grenoble INP, SIMaP, F-38000 Grenoble, France

\# Corresponding Author:

Email: guilhem.martin@ simap.grenoble-inp.fr

Phone: +33 (0)476826342

\begin{abstract}
The possibility to produce Ni-base superalloy single crystals by selective electron beam melting (S-EBM) is demonstrated. The production of single crystals specimens was achieved by a tight control of the processing conditions without requiring a grain selector or a crystal seed. The melting parameters are controlled so as to promote columnar grains and intensify the competitive grain growth.
\end{abstract}

Keywords: Electron Beam Melting; Ni-base superalloys; Single Crystal; Grain selection; Crack-free

Additive manufacturing (AM) is a breakthrough for the production of complex components providing a high degree of freedom in the design of parts and offering the possibility to tailor the microstructure. Since the pioneering work of Hunt $[1,2]$, the magnitude and direction of the thermal gradient as well as the solidification velocity are the keys parameters to modify the solidification structure. In the literature of S-EBM (Selective Electron Beam Melting), it is suggested that changes of the melting strategies enable to alter the thermal gradient and solidification velocity to achieve different grain structures and texture. Helmer et al. $[3,4]$ reported that grain size and morphology can be controlled. Dehoff et al. $[5,6]$ have demonstrated that spatially controlled texture within an Inconel 718 part can be achieved. Such microstructure engineering approach was further investigated by Helmer $e t$ al. [7] and Raghavan et al. [8, 9]. They showed that different scanning strategies enable to control the thermal gradient magnitude and direction resulting in different grain morphologies and sizes. Processing non-weldable Ni-based superalloys by S-EBM appears much more challenging as severe hot cracks may develop [10-12]. Ramsperger et al. have reported the possibility to produce crack-free specimens of CMSX-4 [10]. A recent investigation focused on the mechanism at the origin of the development of hot cracks in a non-weldable Ni-based superalloy fabricated by S-EBM showed that only High Angle Grain Boundaries (HAGBs) are affected by cracks while the Low Angle Grain Boundaries (LAGBs) remain systematically uncracked [12]. This suggests that if one succeeds to tailor the microstructure so as to remove any HAGB, i.e. to generate a single crystal (SX), crack-free samples can be produced. Moreover, SXs are the most efficient way to increase the creep behaviour of Nibased superalloys in the high temperature regime.

Achieving SX through AM is challenging. The literature dealing with the repair of SX components by AM processes is limited [13-15]. Selective Laser Epitaxy (SLE) or Direct Metal Deposition (DMD) were the first AM processes allowing the deposition of several SX layers on the CMSX-4 [14, 15] or René N5 alloys [13]. If one considers the possibility to produce bulk SX using AM processing routes, the literature becomes even poorer. The 
possibility to generate bulk CMSX-4 single crystals by S-EBM was only reported in a conference paper by Ramsperger et al. [16].

In the present contribution, thanks to a tight control of processing parameters and strategy, crack-free bulk single crystals of a non-weldable Ni-based superalloy are produced by S-EBM without requiring a crystal selector or a seed.

The studied alloy is a non weldable Ni-Co-Cr-Mo-Al-Ti-B Ni-based superalloy containing significant amounts of $\mathrm{Cr}$, $\mathrm{Co}$ and $\mathrm{Mo}$ with $\mathrm{Ti}+\mathrm{Al}$ wt. $\%=8.6$. The exact chemical composition cannot be indicated for industrial confidentiality reasons. The prealloyed powder was produced by gas-atomization and provided by ERASTEEL. The asreceived powder particles exhibit mostly a spherical morphology with some satellites $\left(D_{50}=\right.$ $77 \mu \mathrm{m}$ and powder bed relative density $=53.6 \%$, see [12] for more details).

The prealloyed powders were used into an ARCAM A1 EBM machine operating at $60 \mathrm{kV}$ accelerating voltage under a controlled pressure of $2.10^{-3}$ mbar. The powder was deposited by $50 \mu \mathrm{m}$ thick layers on a stainless steel plate, then slightly sintered with a defocused beam and finally selectively melted according to the input CAD geometry. The operating temperature was $\sim 1020^{\circ} \mathrm{C}$. Samples with dimensions of $23 \times 23 \times 30 \mathrm{~mm}$ melted in manual mode, i.e. with the automatic ARCAM functions disabled, were fabricated by varying the scan speed ( $\mathrm{v}$ $=500-1500 \mathrm{~mm} / \mathrm{s})$ and beam power $(\mathrm{P}=225-600 \mathrm{~W})$. Such a processing window was chosen to ensure a high bulk density (>99.5\%, no lack-of-fusion defects) while avoiding significant overmelting leading to uneven surfaces $[3,17]$. This was achieved by using linear energies $\left(\mathrm{E}_{1}\right.$ $=\mathrm{P} / \mathrm{v})$ between 0.3 and $0.6 \mathrm{~J} / \mathrm{mm}$. The melting strategy and focus offset $(20 \mathrm{~mA})$ were held invariants. The melting strategy consists of scanning the area defined by the CAD file in a snake-line way with a line offset of $0.1 \mathrm{~mm}$ and a line order set to 1 . The scanning direction was rotated by $90^{\circ}$ after every layer. The building time for one layer was about $160 \mathrm{~s}$ for a build consisting of 9 samples.

The microstructures were characterized by optical microscopy, SEM (ZEISS Gemini SEM500) and EBSD along the longitudinal cross sections (XZ plane). The microstructures were revealed using a fresh Glyceregia etchant [12]. EBSD measurements were performed using the OIM-TSL software.

A typical as-built sample is shown in Figure 1a. The observations were done in the $\mathrm{XZ}$ plane, see Figure 1a. Figure 1b shows an example of the region located just below the top surface. Figure 1b is analogous to the typical microstructure of a weld since the melted zone, the heat affected zone and the "base material" (built material unaffected thermally by the melted zone) can be distinguished. The terminology "base material" might be excessive here as it can be thermally affected but only during the preheating stages. The height of the last melted zone (depth of the melt pool) can be estimated based on the microstructural characterization performed in the top region, see example in Figure 1b. High power combined with slow scanning speed, corresponding to the highest linear energy $\left(E_{1}=0.6\right.$ $\mathrm{J} / \mathrm{mm}$ ), lead to the deepest melt pools. As expected, for a given power, the melt pool depth increases when the scanning speed decreases. Similarly, for a given scanning speed, the melt pool depth increases with power. Within the processing window selected for a $50 \mu \mathrm{m}$ layer 
thickness, melting occurs from 3 up to 12 layers down corresponding to melt pool depths varying between $150 \mu \mathrm{m}\left(\mathrm{E}_{1}=0.3 \mathrm{~J} / \mathrm{mm}\right)$ and $600 \mu \mathrm{m}\left(\mathrm{E}_{1}=0.6 \mathrm{~J} / \mathrm{mm}\right)$.

The region just below the top surface is of particular interest because it is the only region where the dendritic microstructure inherited from the solidification can be observed. Below this region, the dendritic microstructure is partly or even completely erased by the fact that the underlying layers have been significantly thermally affected during the addition of new layers. It was thought that this rather complex thermal path undergone by the underneath layers could be seen as a sort of solutionizing heat treatment erasing the dendritic microstructure and changing drastically the precipitation state as shown in [12]. Primary dendrite trunks can be easily seen in this last melted zone as shown in Figure 1c. Primary dendrite arm spacing (PDAS), estimated by the intercept method, was found to vary between $\sim 10$ and $30 \mu \mathrm{m}$ depending on the linear energy. The highest linear energy $\left(\mathrm{E}_{1}=0.6 \mathrm{~J} / \mathrm{mm}\right)$ within the identified processing window gives the highest PDAS $(30 \mu \mathrm{m})$. Such PDAS values can be used to infer information regarding the cooling rate from literature data correlating PDAS with cooling rate for several Ni-based superalloys [18]. A PDAS of $10 \mu \mathrm{m}$ suggests a cooling rate of the order of $\sim 500^{\circ} \mathrm{C} / \mathrm{s}$ while a PDAS of $30 \mu \mathrm{m}$ corresponds to a cooling rate of $\sim 30^{\circ} \mathrm{C} / \mathrm{s}$ (one order of magnitude smaller) [18]. PDAS values with the same order of magnitude though a bit smaller (between 3 and $10 \mu \mathrm{m}$ ) were reported recently for Inconel 718 [9]. This is consistent with the localized melt-scan strategy used in [9] leading to higher cooling rates whereas a raster melt-scan strategy was used here.

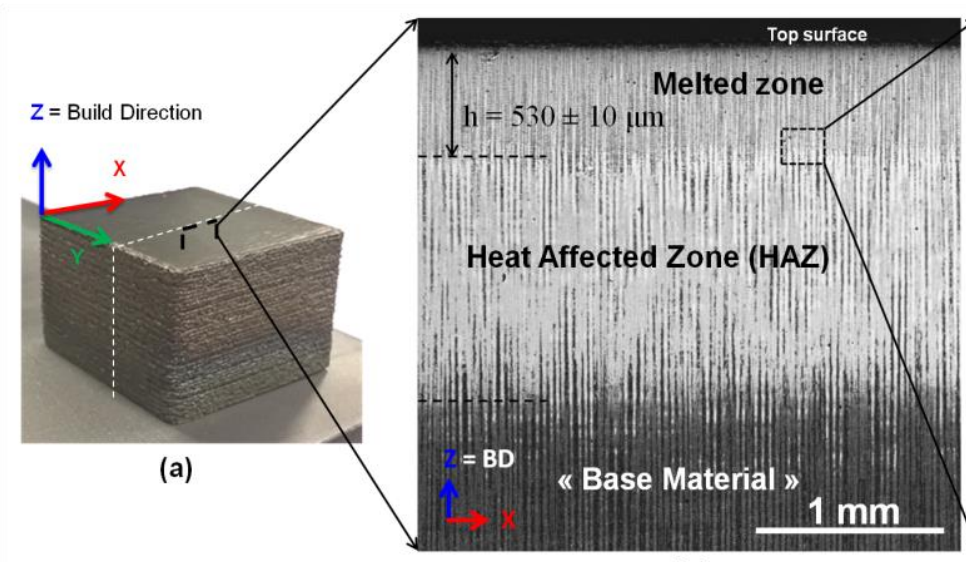

(b)

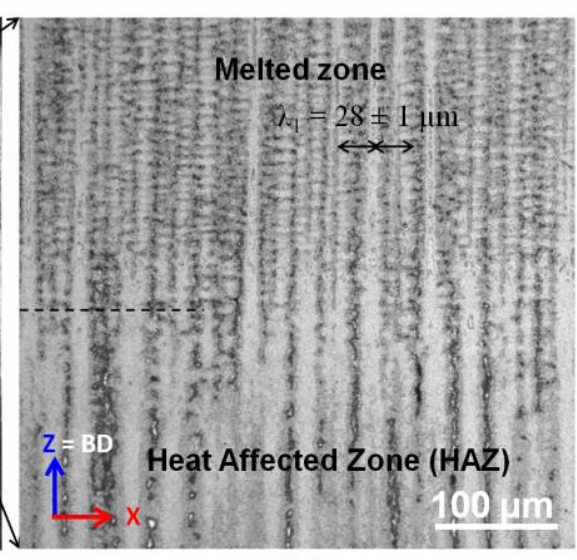

(c)

Figure 1. (a) Typical S-EBM as-built sample. Optical micrographs showing (b) an example of the region located just below the top surface and (c) the dendritic microstructure of the last melted layer.

Microstructural observations in the entire XZ cross section reveal typical features of parts manufactured by AM. The edges of the samples were more or less affected by the local thermal conditions since in those regions, heat was not only extracted by the built material but also by the surrounding powder bed. In this contribution, the attention is focused on the central region of the samples. Melting parameters leading to deep melt pools were deliberately selected to promote epitaxial growth along the building direction. Columnar grains growing epitaxially along the build direction across several layers were found in the central region, see Figure 2a-c. Those columnar microstructures are typical of directionally solidified materials, see e.g. [19, 20]. 
Over the first millimeters, all samples exhibit a similar microstructure consisting of relatively narrow columnar grains (region A in Figure 2a-c). With further addition of layers, texture develops and becomes strongly oriented with a [001]-fiber texture and larger columnar grains grow [12]. The main microstructural differences between the samples are (i) the columnar grain width and (ii) the selection of grain orientation which is more or less pronounced depending on the processing parameters. Figure 2a shows a sample exhibiting an homogeneous microstructure with relatively fine columnar grains (grain width $\sim 100 \mu \mathrm{m}$ ). This sample was melted using a linear energy of $0.3 \mathrm{~J} / \mathrm{mm}$. The associated melt pool depth was found to be $150 \pm 10 \mu \mathrm{m}$ and the PDAS, estimated to $13 \pm 1 \mu \mathrm{m}$. In Figure $2 \mathbf{b}$ is shown a sample and exhibiting a gradient of columnar grain width, their width being close to one millimeter in the upper part (Figure 2b). The melt pool depth was about $260 \pm 10 \mu \mathrm{m}$ and the PDAS about $16 \pm 1 \mu \mathrm{m}$. Figure 2c, melted with a linear energy of $0.45 \mathrm{~J} / \mathrm{mm}$, shows another example where the gradient of columnar grain width was strengthened (columnar grain width of several millimeters in the upper part) using parameters leading to a melt pool depth of 366 $\pm 10 \mu \mathrm{m}$ and a PDAS of $23 \pm 1 \mu \mathrm{m}$. Those observations suggest that with appropriate changes of the processing parameters, the gradient of columnar grain width can be exacerbated by intensifying the grain growth competition until obtaining a single grain orientation after less than $10 \mathrm{~mm}$ of built material. This was achieved when using a linear energy $0.6 \mathrm{~J} / \mathrm{mm}$ leading to a melt pool depth of $532 \pm 10 \mu \mathrm{m}$ and a PDAS close to $30 \mu \mathrm{m}$, see Figure 3a where no grain boundaries can be seen in the upper central part. To summarize, within the processing windows investigated here, the growth of a single crystal is promoted by using high linear energies (typically $0.6 \mathrm{~J} / \mathrm{mm}$ ) to (i) promote epitaxial growth through deep melt pools and (ii) achieve relatively slow cooling rates (highest PDAS) for the S-EBM process to instigate the grain growth selection mechanism while ensuring a high bulk density $\left(\mathrm{E}_{1}<0.3 \mathrm{~J} / \mathrm{mm}\right)$ and avoiding overmelting $\left(\mathrm{E}_{1}>0.6 \mathrm{~J} / \mathrm{mm}\right)$.

To demonstrate that this region is indeed a single crystal, EBSD measurements were performed on different XZ cross sections. IPF-maps presented in Figure 3b-d clearly show the presence of a single crystal. The texture plots displayed in Figure 3c show that a single orientation is present. This single crystal sample was found to be crack-free confirming that removing HAGBs is a solution to suppress cracks [12].

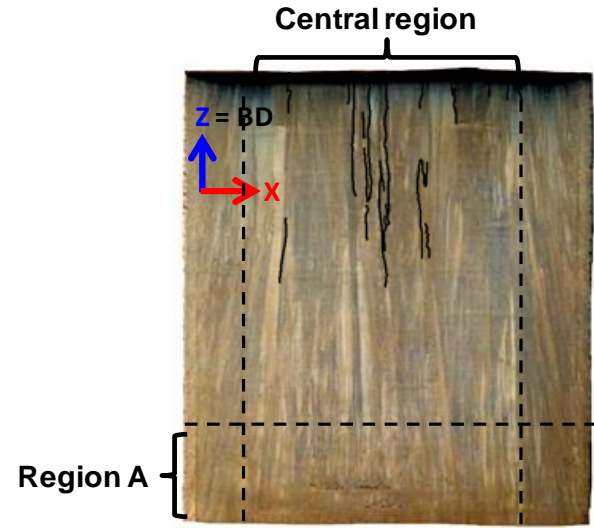

(a)

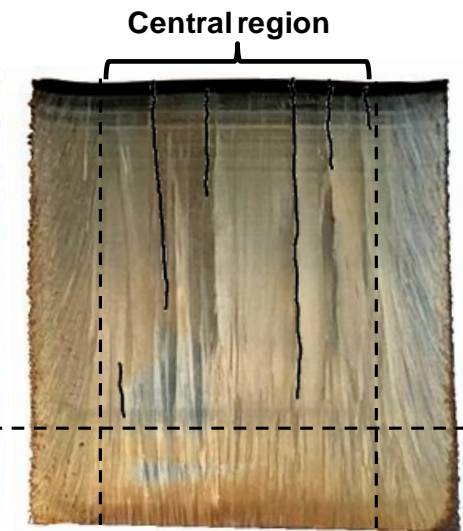

(b)

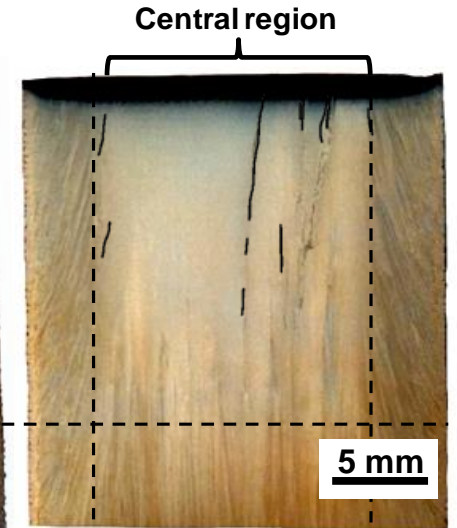

(c)

Figure 2. Optical micrographs showing examples of the grain structures obtained in manufactured samples. (a) Homogeneous microstructure with narrow columnar grains $(\sim 100 \mu \mathrm{m})$. (b) and (c) Graded microstructures with columnar grains of about $1 \mathrm{~mm}$ and several millimeters respectively in width in the upper part. Cracks are highlighted in black. 


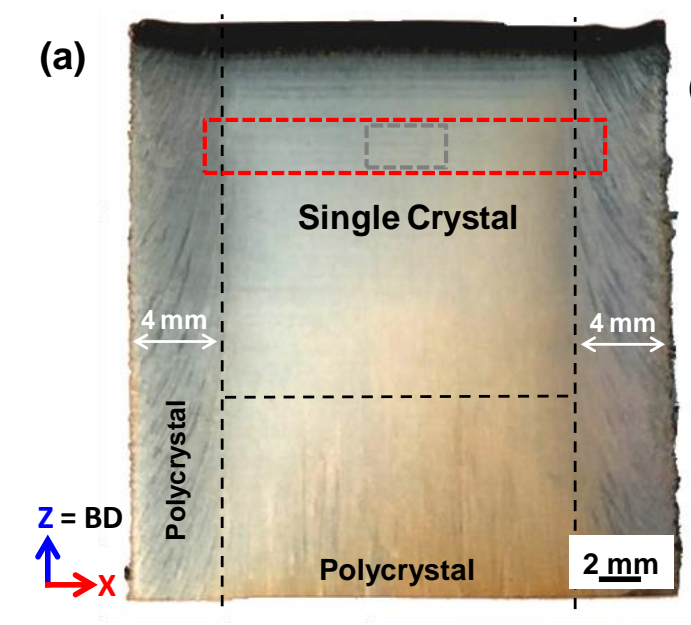

(b)


(d)

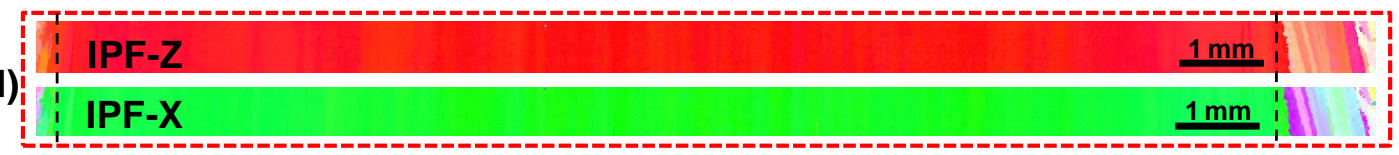

Figure 3. (a) Sample exhibiting a monocrystalline central region. (b) IPF-Z map acquired in the central part. (c) (001) and (111) Pole Figures showing the orientation of the single crystal in the upper part. (d) IPF maps of the whole transverse section demonstrating the presence of a single crystal over a width of more than $15 \mathrm{~mm}$.

To get insights into the mechanism of grain orientation selection leading to a single orientation, additional EBSD measurements were performed in the transition region between the polycrystalline region and the single crystal one, see Figure 4. Two regions can be distinguished in Figure 4a-b: the lower region consists of several columnar grains whereas the upper part consists of a single orientation. In the lower part, the columnar grains are strongly textured, their [001] direction being almost parallel to the building direction (Figure 4b). This is consistent with the fact that in FCC metals, the preferential grain growth directions are the $\langle 001\rangle$ directions. Here, only the [001] direction develops because it corresponds to the direction of the main thermal gradient. However, there are significant misorientations in the XY plane as indicated by the IPF-X map in Figure 4a (two IPF maps are required to fully describe a crystallographic orientation). This is further illustrated by the IPF-Z map displayed in Figure $\mathbf{4} \mathbf{c}$ where all the grains having a misorientation smaller than $15^{\circ}$ respect to the single crystal orientation are highlighted in blue. When the misorientation angle respect to the single orientation is set to $2^{\circ}$, fewer grains are highlighted in blue, see Figure 4d. The upper part is easier to describe since it consists of a single orientation with the building direction and X-direction aligned respectively with the [001] and [110] directions. The selection of a single grain orientation occurs without the requirement of a single crystal start plate acting as a seed or a crystal selector. From the first few layers, a competitive grain growth occurs and intensifies leading to the disappearance of the misoriented grains, i.e. those having their [001] direction not perfectly aligned with the direction of the main thermal gradient. As a result, a strong [001]-fiber texture develops because grains even slightly misoriented respect to the [001] direction tend to disappear in favor of the perfectly aligned ones. If a strong [001]-fiber texture develops, it is not enough to account for the selection of a single orientation. Indeed, grains can be oriented such as having their [001] direction aligned 
with the building direction while being rotated around the [001] direction in the (XY) plane giving large misorientation, see typically the bottom region of Figure 4a. With the addition of new layers, the competitive grain growth is exacerbated until a single orientation grows at the expense of the others. The orientation corresponding (i) to the alignment of the [001] direction along the building direction and (ii) to the alignment of the [110] direction along the $\mathrm{X}$ direction grows at the expense of the others, see Figure 4a-b. The fact that the single crystal has its [110]-direction aligned with the X-direction (one of the scanning direction), is not yet elucidated though it is suspected to be related to the $90^{\circ}$ rotation of the scanning direction after every layer. In the conventional processes using a seed, a single orientation is grown epitaxially. In the single crystals produced here, in the first few layers, there are few grains possessing the [001]-direction perfectly aligned with the Z-direction and the [110] direction perfectly aligned with the X-direction. Those grains with a very similar orientation grow at the expense of the other, and coalesce leading to a $2 \mathrm{~cm}^{3}$ single crystal (Figure $4 \mathbf{c}-\mathbf{d}$ ).

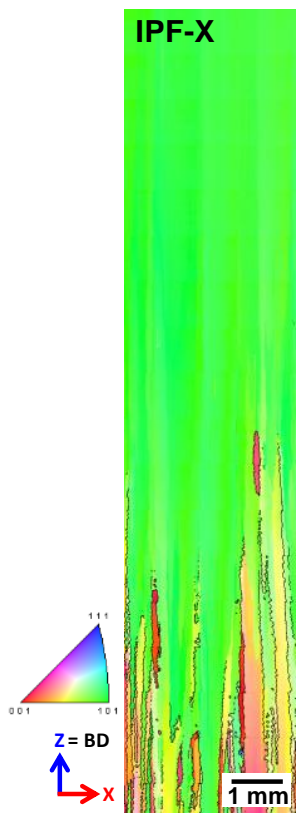

(a)

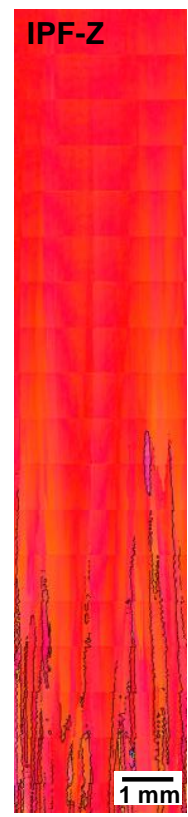

(b)

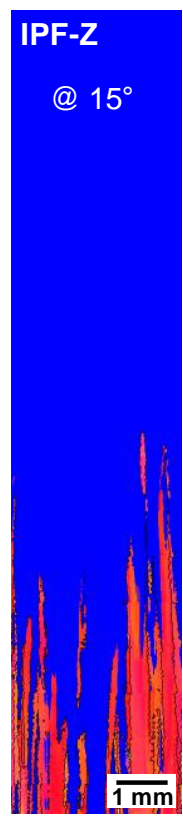

(c)

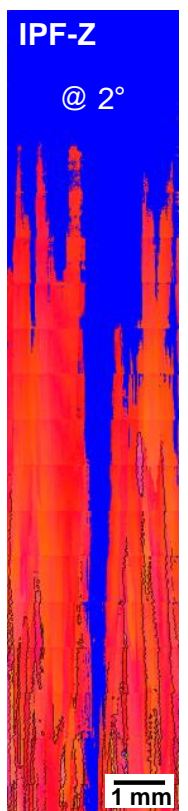

(d)

Figure 4. Transition region between the polycrystalline region (bottom of the sample) and the single crystalline region (top of the sample). (a) IPF-X map. (b) IPF-Z map. (c), and (d) IPF-Z maps with highlighted in blue the orientation of the single crystal respectively with $15^{\circ}$ and $2^{\circ}$ of misorientation. Misorientation of HAGBs $>15^{\circ}$ are highlighted in black.

To ensure the reproducibility of the single crystal growth, another build was run with similar geometry, preheating temperature and melting strategy. Single crystals were successfully grown showing the consistency of the identified melting parameters and strategy. Nevertheless, a change of the preheating temperature or geometry of the samples, would likely require changes in melting parameters or strategy to maintain favorable conditions for single crystal growth, meaning that depending on the geometry of the parts, it might be very challenging to preserve the conditions to grow single crystals.

The results show that within the processing windows investigated, we are clearly in a columnar growth regime where the competitive grain growth can be manipulated. Interpretation of the above-described grain structures can rely on the analogies between $\mathrm{S}$ $\mathrm{EBM}$ and directional solidification such as Bridgman crystal growth. Indeed, the melted zone 
shown in Figure 1b can be assimilated to the melted zone of the ingot in the Bridgman furnace while the underlying layers in the central part of built material act as the cold zone in the Bridgman process, extracting heat in the opposite direction to the growth direction. Besides, at the end of each layer, the build plate goes down by a $50 \mu \mathrm{m}$-increment corresponding to the layer thickness. The layer time being constant throughout the build, the build plate down-velocity is constant similarly to the pulling direction in the Bridgman process.

Identifying the reasons why some processing conditions lead to a single crystal and others not is not an easy task and would require simulations including thermal considerations, melt pool dynamics and morphology as well as local solidification conditions. However, based on the present results, one can give some insights into the effect of some physical parameters on the grain selection mechanism. First, the effect of the melt pool geometry on the dendritic grain growth direction can be invoked. This has been investigated by Liu et al. $[21,22]$. The authors have established some links between the melt pool geometry and the number of selected $\langle 001\rangle$ growth variants and to the relative size of each growth region. Here, the situation is more complicated because the melt pool geometry is not only affected by the melting parameters (beam power, velocity and linear energy) but also by the melting strategy. Moreover, high cooling rates are favorable towards fine grains unlike slower cooling rates that results in larger grains. This is consistent with our results since the samples with the largest grain width are those which were cooled with the slowest cooling rate, as shown by the PDAS measurements. To a certain extent, it indicates that tuning the melting parameters enables to control the solidification conditions (thermal gradient and growth rate) and consequently the location of the transition between a polycrystalline and monocrystalline region along the building direction.

A tight control of the melting parameters enables to produce bulk single crystals by SEBM. This does not require the use of a crystal selector or a seed since melting parameters leading to the intensification of the competitive grain growth turns out to be enough.

\section{Acknowledgements}

This work was performed within the framework of the Center of Excellence of Multifunctional Architectured Materials "CEMAM" n'AN-10-LABX-44-01 funded by the "Investments for the Future Program". The authors are grateful to Poly-Shape for their contribution to funding this work. 


\section{References}

[1] S.C. Flood, J.D. Hunt, Journal of Crystal Growth 82(3) (1987) 552-560.

[2] J.D. Hunt, Materials Science and Engineering 65(1) (1984) 75-83.

[3] H.E. Helmer, C. Koerner, R.F. Singer, Journal of Materials Research 29(17) (2014) 1987-1996.

[4] C. Koerner, H. Helmer, A. Bauereiss, R.F. Singer, in: J.Y. Guedou, J. Chone (Eds.),

Eurosuperalloys 2014 - 2nd European Symposium on Superalloys and Their Applications2014.

[5] R.R. Dehoff, M.M. Kirka, F.A. List, K.A. Unocic, W.J. Sames, Materials Science and Technology 31(8) (2015) 939-944.

[6] R.R. Dehoff, M.M. Kirka, W.J. Sames, H. Bilheux, A.S. Tremsin, L.E. Lowe, S.S. Babu, Materials Science and Technology 31(8) (2015) 931-938.

[7] H. Helmer, A. Bauereiss, R.F. Singer, C. Korner, Materials Science and Engineering a-Structural Materials Properties Microstructure and Processing 668 (2016) 180-187.

[8] N. Raghavan, R. Dehoff, S. Pannala, S. Simunovic, M. Kirka, J. Turner, N. Carlson, S.S. Babu, Acta Materialia 112 (2016) 303-314.

[9] N. Raghavan, S. Simunovic, R. Dehoff, A. Plotkowski, J. Turner, M. Kirka, S. Babu, Acta Materialia 140 (2017) 375-387.

[10] M. Ramsperger, R.F. Singer, C. Korner, Metallurgical and Materials Transactions A-Physical Metallurgy and Materials Science 47A(3) (2016) 1469-1480.

[11] B. Ruttert, M. Ramsperger, L.M. Roncery, I. Lopez-Galilea, C. Korner, W. Theisen, Materials \& Design 110 (2016) 720-727.

[12] E. Chauvet, P. Kontis, E.A. Jägle, B. Gault, D. Raabe, C. Tassin, J.-J. Blandin, R. Dendievel, B. Vayre, S. Abed, G. Martin, Acta Materialia 142 (2018) 82-94.

[13] A. Basak, S. Das, Advanced Engineering Materials 19(3) (2017).

[14] M. Gaumann, C. Bezencon, P. Canalis, W. Kurz, Acta Materialia 49(6) (2001) 1051-1062.

[15] S. Mokadem, C. Bezencon, A. Hauert, A. Jacot, W. Kurz, Metallurgical and Materials

Transactions a-Physical Metallurgy and Materials Science 38A(7) (2007) 1500-1510.

[16] M. Rampsberger, C. Korner, Electron Beam Melting of the single crystalline Ni-base superalloy

CMSX-4: from columnar grains to single crystal, Superalloys 2016, Seven Springs, Pennsylvania,

USA, 2016, pp. 341-349.

[17] E. Chauvet, G. Martin, B. Vayre, S. Abed, R. Dendievel, J.J. Blandin, Additive Manufacturing of Inconel 625 Superalloy Producedby Electron Beam Melting, WorldPM2016, 2016.

[18] H.S. Whitesell, L. Li, R.A. Overfelt, Metallurgical and Materials Transactions B-Process

Metallurgy and Materials Processing Science 31(3) (2000) 546-551.

[19] S. Sun, PhD Thesis, Tohoku University (2015).

[20] S-H. Sun, Y. Koizmi, S. Kurosu, Y-P. Li, H. Matsumoto, A. Chiba, Acta Materialia 64 (2014) 154-168.

[21] W.P. Liu, J.N. DuPont, Acta Materialia 52(16) (2004) 4833-4847.

[22] W.P. Liu, J.N. DuPont, Acta Materialia 53(5) (2005) 1545-1558. 